\title{
Graduate Students and Scholars Funded
}

$\mathrm{T}$ o increase graduate student participation in the Annual Meeting, the association awarded Graduate Student Travel Grants for the 2017 Annual Meeting in San Francisco, California. The travel grants subsidize the cost of travelling to the meeting, and candidates are awarded the grants after applying during the summer months. Every year, Cambridge University Press provides a generous sponsorship to the program as well. APSA was glad to welcome the following graduate students and scholars to the 2017 Annual Meeting:

\begin{tabular}{|c|c|}
\hline INTERNATIONAL GRADUATE STUDENTS & Sergey Sanovich \\
\hline Yehonatan Abramson & Asilhan Saygili \\
\hline Elizabeth Acorn & Eduardo Schmidt Passos \\
\hline Therese Anders & Orcun Selcuk \\
\hline Eric Arias & Gyu Sang Shim \\
\hline Sergio Ascencio Bonfil & Enrijeta Shino \\
\hline Alejandro Beltran & Jungmin Song \\
\hline Joan Carreras Timoneda & Melanie Sonntag \\
\hline Chia-Chien Chang & Dawid Tatarczyk \\
\hline Sinan Chu & Fnu Testriono \\
\hline Menevis Cilizoglu & Sarina Theys \\
\hline Wenhui Feng & Bashir Tofangsazi \\
\hline Jose Antonio Fortou Reyes & Efe Tokdemir \\
\hline Olga Gerasimenko & Shritha Vasudevan \\
\hline Lara-Zuzan Golesorkhi & Jelena Vicic \\
\hline Taha Hameduddin & Giancarlo Visconti \\
\hline Jonathan Homola & Tao Wang \\
\hline Han-Hui Hsieh & Yiran Wang \\
\hline Injeong Hwang & Ezgi Yildiz \\
\hline In Hyee Hwang & Yeo Jung Yoon \\
\hline Filiz Kahraman & Veronica Zebadua Yanez \\
\hline Myunghoon Kang & Zhu Zhang \\
\hline \multicolumn{2}{|l|}{ Jennifer Kartner } \\
\hline Aycan Katitas & INTERNATIONAL SCHOLARS \\
\hline Friederike Kelle & Huda Alsahi \\
\hline Seungjun Kim & Senka Anastasova \\
\hline Jeehye Kim & Merih Angin \\
\hline Ore Koren & Mert Arslanalp \\
\hline Igor Kovac & Su Hyen Bae \\
\hline Alexander Kustov & Sandrine Berges \\
\hline Dongil Lee & Dragana Bodruzic \\
\hline Kyu Young Lee & Juan Bogliaccini \\
\hline Seunghyun Lee & Bjoern Bremer \\
\hline Ashley Lee & Fiona Buckley \\
\hline Ecaterina Locoman & Radzhana Buyantueva \\
\hline Ana Luiza Melo Aranha & Andreia Carmo \\
\hline Simeon Mitropolitski & Cristiane Carneiro \\
\hline Mert Moral & Yajiao Chen \\
\hline Gautam Nair & Yi-Chun Chien \\
\hline Clara Neupert-Wentz & Rebecca Cordell \\
\hline Sinh Nguyen & Cynthia Cunha \\
\hline Yuree Noh & Jean-François Daoust \\
\hline Mary Nugent & Llaria Di Gioia \\
\hline Jiayun Elvin Ong & Ehud Eiran \\
\hline Melda Ozsut & Pilar Elizalde \\
\hline Joanna Phua & Cengiz Erisen \\
\hline Lucas Pinheiro & Pedro Floriano Ribeiro \\
\hline Adnan Rasool & Rosario Forlenza \\
\hline Joan Ricart-Huguet & Yuru Fu \\
\hline Anselm Rink & Michael Gavin \\
\hline
\end{tabular}

Niels Goet

Gerda Hooijer

Christine Huebner

Jacob Kanter

Kerim Kavaklı

Anne-Kathrin Kreft

Adele Lebano

Gjovalin Macaj

Marlene Mauk

Lee Morgenbesser

Jennifer Mussell

Katariina Mustasilta

Robert Nagel

Veronika Patkós

Marina Petrova

Michael Räber

Wojciech Rafalowski

Massimo Ramaioli

Aaron Rapport

Frank Reichert

Lukas Schlogl

Max Skjönsberg

Azer Sumbas

Daniel Troup

Nhu Truong

Isil Turkan Ipek

Anastasia Ushakova

Graziela Vital

Vuk Vukovic

Binfan Wang

Steven Yet

Junyan Zhu

Annette Zimmermann

Claudia Zucca

Aftab Alam

Arsene Brice Bado

Naima Benlarabi

George Bob-Milliar

Tarila Ebiede

Victor Isumonah

Akin Iwilade

Aikande Kwayu

Bamlaku Mengistu

US SCHOLARS

Dawn Brancati

Jeffrey Crouch

Adam Enders

Katherine Goktepe

Catherine Guisan 
Angela Ju

Sigrun Kahl

Jeffrey Karam

John Koehler

Patrick Larue

Mara Marin

Yalidy Matos

Eleonora Mattiacci

Mona Morgan-Collins

Eli Naffah

Elva Orozco

Caleb Pomeroy

Matt Scroggs

Kimberly Shella

Althea Sircar

Rebekah Sterling

Emily Sydnor

Nina Valiquette

Marta Vrbetic

Allison White

Zining Yang

\section{US GRADUATE STUDENTS}

Carolyn Abott

Meir Alkon

Giorleny Altamirano

Alex Antony

Margaret Ariotti

Stephen Bagwell

Amanda Blair

Marjorie Breslawski

Kylee Britzman

Lindsay Burt

Ali Bustamante

Shakari Byerly

Roberto Carlos

Don Casler

Brett Cease

Chinbo Chong

Youssef Chouhoud

Richard Clark

Jennifer Corby

Mia Costa

Charles Crabtree

Royal Cravens

Jonathan Crock

Jesse Crosson

Giuseppe Cumella

Anna Daily

Elaine Denny

Michael Dirksen

Lindsay Dolan
Chelsea Ebin

Andrew Engelhardt

Stephanie Erev

Chris Evans

Bailey Fairbanks

Samuel Frantz

Samantha Godwin

Takiyah Harper

Christopher Harris

Victor Haynes

Timothy Hibbard

Heather Hicks

Agatha Hultquist

Deanne Hurtado

Sam Jackson

Nicholas Jacobs

Clinton Jenkins

Tyler Jost

Rafael Khachaturian

Christina Kinane

Austin Knuppe

Tyler Kustra

Kristina Laplant

Danielle Lemi

Scott Limbocker

Erica Macdonald

Emma Mackinnon

Coty Martin

Melissa Martinez

Erika Masaki

Christina Mcelderry

Brandon Merrell

Anna Meyerrose

Timothy Model

Eric Mosinger

Elizabeth Newcomer

Arielle Newman

William Nomikos

William O'Brochta

Angela Ocampo

Christopher Pace

Francesca Parente

Emily Pechar

Fabiana Perera

Isabel Perera

Anthony Pierucci

Jonathan Pinckney

Chase Porter

Abigail Post

Darry Powell-Young

Alireza Raisi

Carrie Reiling
Mara Revkin

Catherine Reyes-Housholder

Samuel Rhodes

Natalie Rogol

Andrew Rosenberg

Tye Rush

Jeheung Ryu

Amy Sentementes

Kelly Senters

William Sisk

Alyssa Sloane

Kelly Smith

Lindsey Smith

Steven Sparks

Andrew Stone

Logan Strother

Clara Suong

Ryan Tans

Rebecca Tapscott

Ezra Temko

Savannah Titus

Mark Toukan

Dilara Uskup

Eric Van Der Vort

Everett A. Vieira, III

Jacqueline Vimo

Philip Waggoner

Kathryn Wainfan

David Walsh

Simon Williamson

Catherine Wineinger

Daniel Wollrich

Adam Wunische

Amanda Zadorian

Matthew Zarit

FUND FOR LATINO SCHOLARSHIP

Rudy Alamillo

Teresa Yolotl Gomez

MINORITY FELLOWSHIP PROGRAM

Elias Assaf

Shakari Byerly

Chinbo Chong

Gregory Leslie

Soledad Prillaman

Amber Spry

Tye Rush

Patricia Posey

Christine Slaughter

Juan Tellez 